\title{
EFFECT OF CRYSTAL ROTATION ON EARING IN CUP DRAWING OF HIGHLY TEXTURED MATERIALS
}

\author{
K. C. CHAN \\ Department of Manufacturing Engineering, The Hong Kong Polytechnic University, \\ Hung Hom, Hong Kong
}

(Received 10 September 1995)

\begin{abstract}
In this paper, a numerical model taking into account of crystal rotation is presented to predict earing in cup drawing. The model is based on the Continuum Mechanics of Textured Polycrystals and the assumption of plane strain. Ear profiles have been predicted for some common crystallographic orientations in face-centered cubic metals and highly textured sheets. It was shown that the major trends of the predicted profiles for both the single crystals and highly textured sheets were consistent with experimental results. The effect of crystal rotation on earing was shown to be insignificant for the predicted ear profiles. However, a more sophisticated model with the consideration of the changes of both texture and stress states should be used for more accurate predictions.
\end{abstract}

KEY WORDS: Earing texture, crystal rotation, sheet metals and deep drawing.

\section{INTRODUCTION}

Earing which leads to nonuniform cup height is of a great concern in the deep drawing of metal sheets. The cups have to be trimmed to produce the correct container height. It obviously involves an extra operation and creates additional scrap. Many attempts have been made to predict earing. Hill (1950) first proposed an explanation for earing based on his anisotropic yield function. Sowerby and Johnson (1974) used Hill's yield function with the plane strain assumption to predict earing. Finite element method (Saran and Samuelsson, 1989; Becker et al., 1992) has also been adopted to simulate earing. In their models, the material anisotropy is often incorporated through a macroscopic anisotropic yield surface. Although the models based on these yield functions are more computational efficient, current macroscopic yield surfaces are criticised for being unable to characterise the complete shape and size of the actual yield surface and to consider the microstructure of metals.

Some models based on the plastic slip have been developed in order to take into account of microstructure of metals. Based on the Schmid law Tucker (1961) has proposed a model to predict face-centered cubic single crystals. Kanetake et al. (1983) has applied Tucker's work to the polycrystal case. Numerous studies have also used the Bishop and Hill polycrystal model (1951) and the orientation distribution functions (ODFs) of textured materials to predict earing (Da Costa Viana et al., 1978; Rodrigues and Bate, 1985; Van Houtte et al., 1987). On the other hand, Lin et al. (1991) have adopted the Continuum Mechanics of Textured Polycrystals (CMTP) method and the assumption of plane stress to predict earing. The CMTP method provides a connection 
between the texture and the plastic anisotropy of the material. However, the predictions were shown to be inconsistent with the experimental results for some crystallographic orientations. The plane stress assumption for earing prediction has been commented by Barlat et al. (1991). They proposed a model of flange behaviour during cup drawing using plane stress or plane strain assumption and concluded that the plane strain assumption for the flange behaviour successfully explained the major trends for earing of both the single crystal and the high-purity aluminium sheets. A numerical model based on the CMTP method and the assumption of plane strain has been proposed by the author. Improvement has been reported as compared with the model of Lin et al. In the present paper, the model is further extended to incorporate with crystal rotation to study the effect of the change of crystallographic texture on earing.

\section{MODEL}

The present model is basically an extension of the model previously proposed by the author. Some essential parts of the proposed model are still outlined in this paper to keep it self-consistent. During the deep drawing of a circular blank, the radial stressed are tensile whereas the circumferential stresses are compressive at all points in the flange. The corresponding strains are compressive along the circumferential direction and tensile strain along the radial direction. In this paper, we assume a series of element in the flange and the assumption of no thickening of the blank (plane strain) is based, i.e., the two strains are equal. The strain increment and true stress tensors for a element are represented by

$$
\begin{aligned}
& d \varepsilon=d \varepsilon_{\theta}\left[\begin{array}{ccc}
1 & 0 & 0 \\
0 & -1 & 0 \\
0 & 0 & 0
\end{array}\right] \\
& \sigma=\left[\begin{array}{ccc}
\sigma_{\mathrm{r}} & \sigma_{\mathrm{r} \theta} & 0 \\
\sigma_{\mathrm{r} \theta} & \sigma_{\theta} & 0 \\
0 & 0 & \sigma_{\mathrm{t}}
\end{array}\right]
\end{aligned}
$$

where $d \varepsilon_{\theta}$ is the strain increment, $\sigma_{\mathrm{r}}$ is the stress along the simulated circumferential direction, $\sigma_{\theta}$ is the stress along the circumferential direction, $\sigma_{\mathrm{r} \theta}$ and $\sigma_{\theta \mathrm{r}}$ are the shear stresses. The stress states of each element are determined for each group of grains by applying the flow rule to the anisotropic continuum yield loci of the Continuum Mechanics of Textured Polycrystals (CMTP) as shown below

$$
\begin{aligned}
F(S)= & \alpha\left(\left|S_{11}-S_{22}\right|{ }^{n}+\left|S_{22}-S_{33}\right|{ }^{n}+\left|S_{33}-S_{11}\right|{ }^{n}\right) \\
& +2 \beta\left(\left|S_{12}\right|{ }^{n}+\left|S_{23}\right|{ }^{n}+\left|S_{31}\right|{ }^{n}\right)=\left(\sqrt{6 \tau_{c}}\right)^{n}
\end{aligned}
$$

In this model, the circumferential strain increment is further taken to vary inversely with the circumferential stress as suggested by Lin et al. (1991), as given by

$$
\frac{d \varepsilon_{\theta}}{d \varepsilon_{\theta 1}}=\frac{\sigma_{\theta 1}}{\sigma_{\theta}}
$$

where $d \varepsilon_{\theta 1}$ and $\sigma_{\theta 1}$ are the circumferential strain increment and stress in the element which corresponds to the rolling direction. This relation is based on the observation 
that the local deformations are usually larger in regions where the material yield stresses are lower.

The final cup height $h$ is composed of two parts

$$
h=h_{o}+h_{r}
$$

where $h_{o}$ is the cup height if the radial strain is zero, and $h_{r}$ is the elongation of the flange.

According to Tucker (1961), $h_{o}$ can be obtained by

$$
h_{o}=0.25\left\{4\left(R_{b}-R_{p}\right)+(4-\pi)\left(2 r_{p}+t\right)\right\}
$$

where $R_{b}$ and $R_{p}$ are the radii of the blank and the punch, $r_{p}$ is the radius of the punch profile, and $t$ is the thickness of the blank. $h_{r}$ can be determined by introducing a constant $c_{r}$,

$$
C_{r}=\frac{\bar{h}_{r}}{d \bar{\varepsilon}_{r}}
$$

where $d \varepsilon_{r}$ is the average radial strain increment, and $h_{r}$ is the average elongation of the flange. They are determined by

$$
d \bar{\varepsilon}_{r}=\sum_{i=1}^{m} \frac{\pi}{m} d \varepsilon_{r i}
$$

where $m$ is the number of elements, and

$$
\hbar_{r}=\frac{R_{b}^{2}-R_{p}^{2}}{2 R_{p}}+t-h+0.43 r_{p}
$$

Equation (9) is based on the assumption that the surface area of the cup is approximately the same as that of the blank (Eary and Reed, 1990). If the final elongation of the flange at different locations is assumed to be proportional to the local radial straining $d \varepsilon_{r}$, equation (7) can be developed into

$$
C_{r}=\frac{h_{r}}{d \varepsilon_{r}}
$$

So the final cup height can be calculated from

$$
h=h_{o}+C_{r} d \varepsilon_{r}
$$

After the element has been deformed by a small strain increment, there is a change in the grain orientation which is dependent on the initial crystallographic orientation. For simplicity, Bishop and Hill's principle of maximum work (1951) and Renouard and Wintenberger's method (1981) of minimum second order plastic work are adopted in this paper for the prediction of crystal rotation. After the new orientation has been determined, the cup is further deformed and the final ear profiles are hence determined after each successive incremental step taking into account the crystal rotation. 


\section{RESULTS AND DISCUSSION}

The cup heights predicted by the present model with and without consideration of crystal rotation for some common crystallographic orientations are exhibited. The orientations considered were $\{100\}<001>,\{111\}<110>,\{110\}<001>$ and $\{112\}<011>$. The constants $\mathrm{n}, \alpha$ and $\beta$ used in equation (3) are $1.6,0.46,0.51$ respectively (Lequeu and Jonas, 1988). The geometry of the cupping dies for ideal orientations were chosen from the experiments of Tucker (1961). Predictions of earing for the four crystallographic orientations are performed as shown in Figure 1. The predictions are shown to be consistent with the experimental results which agree with the finding of Barlet et al. Based on the present model, it was found that the effect of change in crystallographic texture during cup drawing is not significant for the predicted ear profiles.
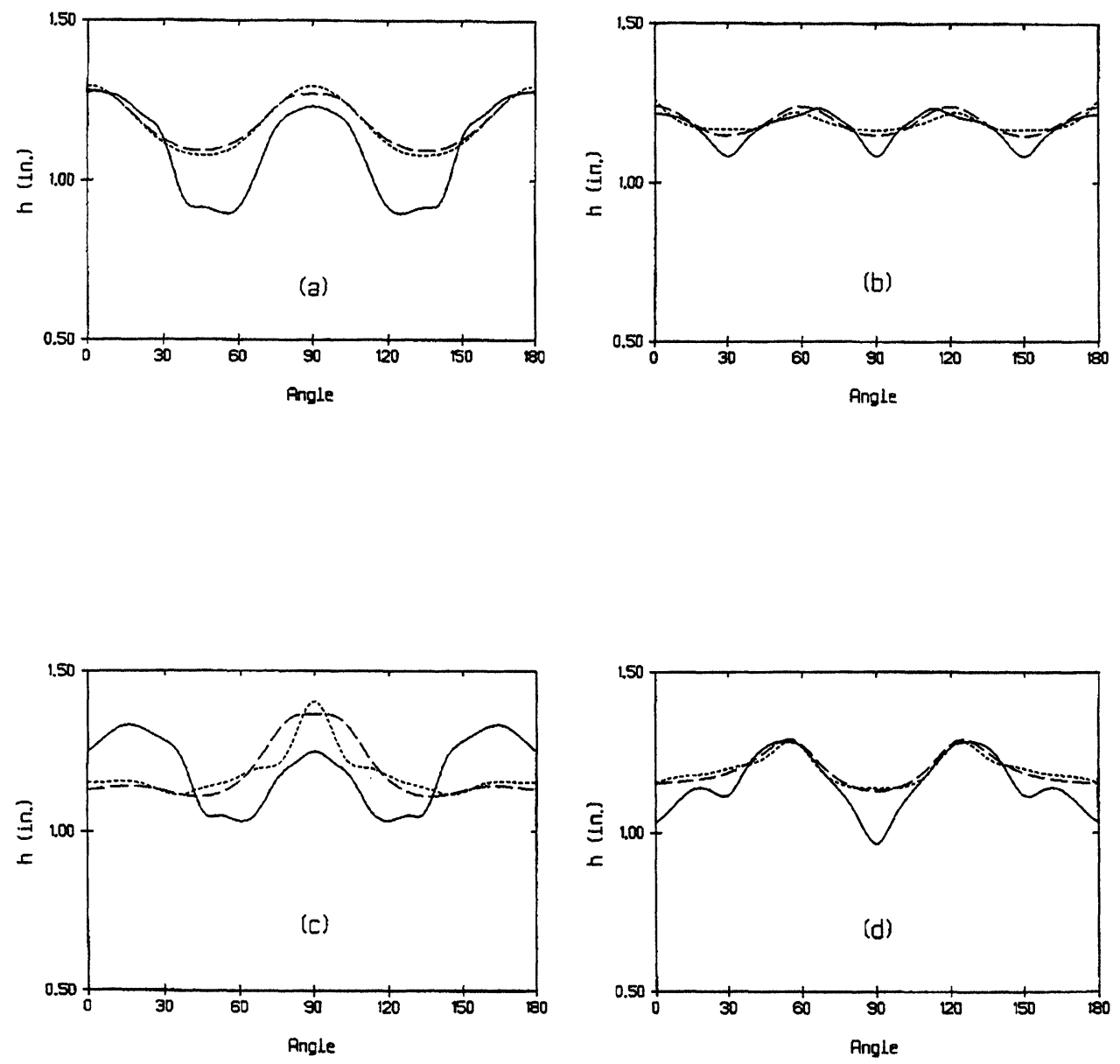

Figure 1 Comparison of predicted cup heights by the present model (i) without considering crystal rotation (- - - ) (ii) taking into account of texture evolution (..........) for a $\{100\}<001>$, (b) $\{111\}<110\rangle$ (c) $\{110\}<001>$ and (d) $\{112\}<110>$ with experimental results $(\longrightarrow)$ taken from ref. 5 . 

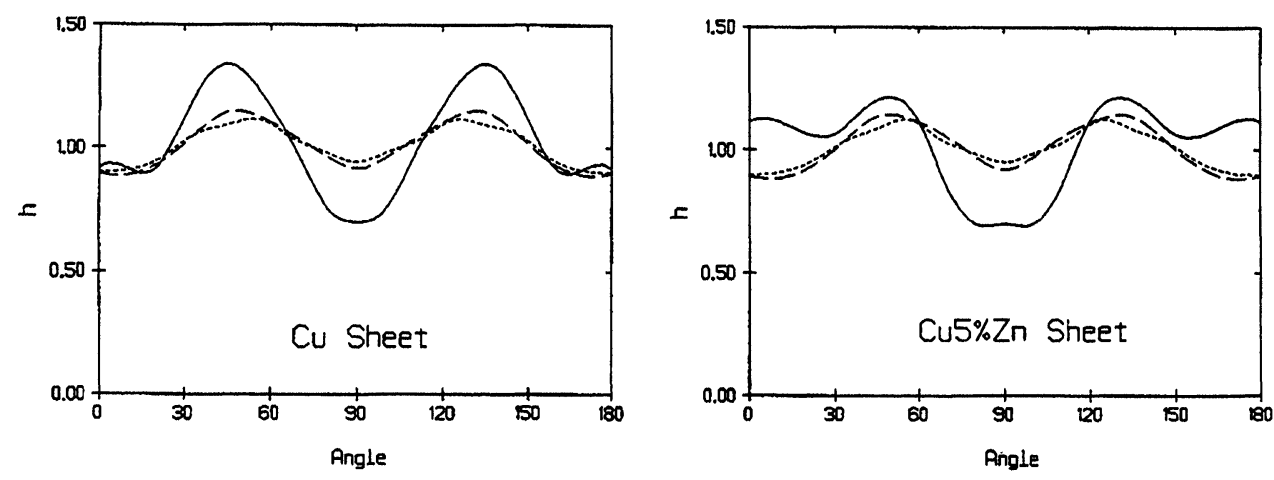

Figure 2 Comparison of predicted cup heights by the present model (i) without considering crystal rotation (- - - ) (ii) taking into account of texture evolution (...........) for textured $\mathrm{Cu}$ and $\mathrm{Cu} 5 \% \mathrm{Zn}$ sheets with experimental results $(-)$ taken from ref. 17.

The present model is also adopted to predict the earing of textured copper and copper zinc alloy sheets. The sheet textures which are represented by $\{110\}<112>(21.96 \%)$ $+\{112\}<111>(29.72 \%)+\{123\}<634>(48.31 \%)$ and $\{110\}<112>(32.81 \%)+$ $\{112\}<111>(21.72 \%)+\{123\}<634>(40.87 \%)+\{110\}<001>(4.6 \%)$ respectively and the geometry of the cupping dies are given by Hirsch et al. (1978). Random orientations are not taken into account in the present analysis. The trend of the predicted ear profiles is consistent with the experimental results as indicated in Figure 2. The effect of crystal rotation on earing is again shown to be insignificant. Since the ratio of the tensileto-compressive stress varies along the radius from zero at the rim of the flange to about two near the edge of die, actual stress states instead of using the assumption of plane strain should be incorporated in the model in order to have a more accurate prediction. However, the present model is shown to be able to predict major ear profiles.

\section{CONCLUSIONS}

In this work, a simplified model based on the CMTP approach has been presented to study the effect of crystal rotation on earing in deep drawing of metal sheets. The earing model has been applied to the case of fcc metals and in agreement with the experimental findings. According to the present analysis, the effect of the change in crystallographic texture for those concerned orientations during cup drawing is found to be insignificant in ear formation. However, for more accurate predictions, a more sophisticated model with the consideration of the change of both texture and stress states should be used.

\section{References}

Barlat, F., Panchanadeeswaran, S. and Richmond, O. (1991). Metal. Trans., A, 22A, 1525.

Becker, R., Smelser, R. E. and Panchanadeeswaran, S. (1992). Computational Methods in Materials Processing, ASME, MD-Vol. 39.

Bishop, J. F. W. and Hill, R. (1951). Phil. Mag., 42, 1298.

Da Costa Viana, C. S., Davis G. J. and Kallend, J. S. (1978). Proc. of the 5th Int. Conf. Texture of Materials, Springer Verlag, Berlin, vol. 2, p. 447. 
Eary, D. F. and Reed, E. A. (1990). Techniques of Pressworking Sheet Metal, Prentice Hall, NJ. Hill, R. (1950). The mathematical Theory of Plasticity, p. 328, Oxford University Press, Oxford.

Hirsch, J., Musick, R. and Lucke, K. (1978). Proc. of the 5th Int. Conf. Texture of Materials, Springer Verlag, Berlin, vol. 2, p. 437.

Kanetake, N., Tozawa, Y. and Otani , T. (1983). Int. J. Mech. Sci., 25, 337.

Lequeu, Ph. and Jonas, J. J. (1988). Metall. Trans, A, 19, 105.

Lin, D. W., Daniel, D. and Jonas, J. J. (1990). Mater. Sci. Eng., A131, 161.

Renouard, M. and Wintenberger, M. C. R. (1981). Acad Sci. Paris Ser., B292, 385.

Rodrigues, P. M. B. and Bate, P. S. (1985). Texture in Non Ferrous Metals and Alloys, TMS, Warrendale, PA, p. 173.

Saran, M. and Samuelsson, A. (1989). NUMIFORM 89, Numerical Methods in Industrial Forming Processes, Balkema, Rotterdam, p. 45.

Sowerby, R. and Johnson, W. J. (1974). Strain Anal., 9, 102.

Tucker, G. E. G. (1961). Acta Metall., 9, 275.

Van Houtte, P. Cauwenberg, G. and Aernoudt, E. (1987). Mater. Sci. Eng., 95, 115. 